\title{
Performance criteria and assessment of vendors in government sponsored entrepreneur development programme
}

\begin{abstract}
In Entrepreneur Development Programme, vendor rating is important not only in supplier selection and in deciding how to allocate business but also determine where scare development effort is best-applied. The paper reviews current methods for vendor rating and finds them wanting. It illustrates a new approach based on the use of Saatyô Analytic Hierarchy process method, which was developed to assist in multi-criteria decision problems. The new method overcomes the difficulties associated with the categorical and simple linear weighted criteria ranking methods. It provides a more systematic way of deriving the weights to he used and for scoring the performance of vendors.
\end{abstract}

Keyword: Vendor rating; Entrepreneur development programme 